Asian J. Med. Biol. Res. 2018, 4 (1), 86-94; doi: 10.3329/ajmbr.v4i1.36826

\author{
Asian Journal of \\ Medical and Biological Research \\ ISSN 2411-4472 (Print) 2412-5571 (Online) \\ www.ebupress.com/journal/ajmbr
}

\title{
Article \\ The effect of the supplementation of lamb feed with Yucca schidigera on the lipid oxidation, color, physico-chemical and microbiological properties in meat
}

\author{
Halit IMIK ${ }^{1}$, Recep GÜMÜŞ ${ }^{2}$, Hayrunnisa ÖZLÜ${ }^{3}$, Sevda URÇAR GELEN ${ }^{3}$ and Meryem AYDEMIR \\ ATASEVER $^{3}$ \\ ${ }^{1}$ Department of Animal Nutrition and Nutritional Disorders, Faculty of Veterinary Medicine, Ataturk \\ University, Erzurum, Turkey \\ ${ }^{2}$ Department of Animal Nutrition and Nutritional Disorders, Faculty of Veterinary Medicine, Cumhuriyet \\ University, Sivas, Turkey \\ ${ }^{3}$ Department of Food Hygiene and Technology, Faculty of Veterinary Medicine, Ataturk University, Erzurum, \\ Turkey
}

*Corresponding author: Sevda URÇAR GELEN, Department of Food Hygiene and Technology, Faculty of Veterinary Medicine, Ataturk University, Erzurum, Turkey. E-mail: surcar@atauni.edu.tr

Received: 07 March 2018/Accepted: 26 March 2018/ Published: 29 March 2018

\begin{abstract}
In this study, the effects of the supplementation of lamb rations with Yucca schidigera powder (YSP) on water activity, $\mathrm{pH}$, lipid peroxidation, oxidation of colour parameters and microbial counts in the Musculus gluteo biceps and Musculus longissimus dorsi were investigated. Three groups were established, namely, the Control group fed on a basal ration and Groups YSPI and YSPII given a basal ration added $100 \mathrm{mg} / \mathrm{kg}$ and 200 $\mathrm{mg} / \mathrm{kg}$ of Yucca schidigera powder, respectively. YSP affected $\mathrm{pH}$ and lipid peroxidation in the gluteo biceps and longissimus dorsi muscles, $\mathrm{L}^{*}$ (Lightness) in the former and $\mathrm{L}^{*}$ and $\mathrm{a}^{*}$ (redness) in the latter. YSP reduced Enterobacteriaceae and total mesophilic aerobic bacteria counts in both muscles, while its effects on total psychrotrophic aerobic bacteria, Micrococcus/Staphylococcus, Staphylococcus aureus, yeast and mold counts varied. Prolonged storage negatively affected the quality of both muscles, but YSP provided significant amelioration. Dietary supplementation with YSP improved meat quality and shelf life.
\end{abstract}

Keywords: antimicrobial; antioksidant; lamb; lipid peroxidation; meat quality; Yucca schidigera

\footnotetext{
1. Introduction

It is well known that nutrition strategies play a significant role in increasing productivity and quality in animal production. Antibiotics have been used extensively for this purpose in animal nutrition for many years. However, upon the reveal of their detrimental effects on human health, their incorporation into feed rations has been prohibited. Thus, vitamins, minerals and plant extracts and powders have started to be used as feed additives and as an alternative to antibiotics. In the food sector, antioxidant additives are used to prevent oxidation reactions, such as the rancidity of fats and colour change, as well as the microbial spoilage of food, and thus, serve to extend the shelf life of food products (Alimentarius, 2002). As a matter of fact, research conducted to date has demonstrated that antioxidant vitamins positively affect both the quality and shelf life of meat (Imik et al., 2010; Imik et al., 2012; Imik et al., 2012 and Oral et al., 2014). Phytochemical substances found in the structure of plants are used in both human medicine for their antioxidant, antimicrobial, antiparasitic, immunostimulatory, anticarcinogenic, anticholesterol and antihypertensive effects, as well as in the food industry as additives (Cheeke, 2009; Piacente, 2004).

Depending on the levels of phenolic compounds and saponins it contains, Yисca is used for its ammoniumbinding, urease-inhibiting, surface-tension reducing (in the intestinal epithelium), antibacterial, antiprotozoal, antifungal and antioxidant effects. It has been reported that, when administered to ruminants and monogastric
} 
animals, it positively affects vigour, nutrient digestion and absorption, and yields (Kutlu et al., 2001). The US Food and Drug Administration (FDA) has reported that products containing Yucca schidigera and Quillaja saponaria are, in general, safe and harmless (Tanaka et al., 1996).

Although Yucca schidigera is used for multiple purposes, no literature report is available on its effects on meat quality when used as a feed additive. In this respect, this study was aimed at determining, in detail, the effect of Yucca schidigera on post-mortem metabolism, which defines meat quality. This study investigated the effects of Yucca schidigera on the water activity, $\mathrm{pH}$ (acidification), lipid peroxidation, colour parameter $\left(\mathrm{L}^{*}, \mathrm{a}^{*}\right.$ and $\left.\mathrm{b}^{*}\right)$ oxidation and microbial properties of the Musculus gluteo biceps and Musculus longissimus dorsi.

\section{Materials and Methods}

\subsection{Animals, experimental design and feed}

The trial was conducted at the premises of the Research and Practice Unit of Ataturk University, Faculty of Veterinary Medicine. Twenty-four 3-month-old lambs of the Akkaraman breed were fed on a basal ration, the composition of which is presented in Table 1, for a period of 70 days. From each group, which reached a mean final body weight of $47 \pm 0.5 \mathrm{~kg}$ at the end of the fattening period, 8 animals, and in total 24 lambs, were slaughtered for the analysis of the gluteo biceps and longissimus dorsi muscles. Of the trial groups, the control group was fed on the basal ration alone, Group YSPI on the basal ration supplemented with $100 \mathrm{ppm}$ of Yucca schidigera powder, and Group YSPII on the basal ration supplemented with $200 \mathrm{ppm}$ of Yucca schidigera powder. This study was approved by the ethics committee of Ataturk University Health Sciences Institute (Decision No: 2010/5.1/16).

\subsection{Yucca schidigera powder (EkomixYucca)}

EkomixYucca, $120 \mathrm{mg}$ Yucca schidigera powder per kg, Ekol Food Agriculture and Livestock Marketing Industry and Trade Inc.

\subsection{Feed analysis}

The analyses of the feedstuffs used in the study for crude protein and crude fat proportions were performed in accordance with the methods laid down by the Association of Official Analytical Chemists (AOAC, 2005), and crude cellulose analyses were performed as described by Van Soest and Robertson (Van Soest and Robertson, 1985).

\subsection{Determination of meat quality}

The animals were slaughtered after being fasted for a period of 10 hours. After the carcasses were maintained at $+4^{\circ} \mathrm{C}$ for 24 hours, the Musculus gluteo biceps (M. superficialis + M. biceps femoris) and Musculus longissimus dorsi were extracted and divided into 7 portions. The extracted muscles were placed into polyethylene plates, covered with stretch film, and stored at $4 \pm 1{ }^{\circ} \mathrm{C}$ during storage period. On 1., 3., 5., 7. and 11. days the meat samples analaysed for water activity, $\mathrm{pH}$, thiobarbituric acid-reactive substances (TBARS) and colour [L*(relative lightness), $a^{*}\left(\right.$ relative redness), $b^{*}$ (relative yellowness)] were analaysed and microbial counts [Enterobacteriaceae, total psychrotrophic aerobic bacteria (TPAB), total mesophilic aerobic bacteria (TMAB), Micrococcus/Staphylococcus, Staphylococcus aureus (S. aureus), yeasts and molds] were performed on the samples. Microbiological analyses of the samples preceded the other analyses.

Water activity values were measured using an Aqualab 4TE (USA) device. Meat samples were placed in the container of the device for the reading of the $\mathrm{a}_{\mathrm{w}}$ values.

The $\mathrm{pH}$ values of the samples were measured as described by Gokalp et al. (2001). Accordingly, 10 g-portions of the homogenized samples were weighed and each portion was added $100 \mathrm{ml}$ of distilled water. Homogenization was performed for 1 minute using an Ultra-Turrax (IKA Werk T 25, Germany) homogenizer and the $\mathrm{pH}$ values were measured using a $\mathrm{pH}$-meter (WTW Inolab, Germany).

For the determination of the TBARS values, $2 \mathrm{~g}$ of the homogenized samples were added $12 \mathrm{ml}$ of TCA solution (7.5\% TCA, $0.1 \%$ EDTA, $0.1 \%$ propyl gallate dissolved in $3 \mathrm{ml}$ ethanol), homogenized for $15-20$ seconds on an Ultra-Turrax homogenizer, and subsequently, filtered through Whatman 1 filter paper. Of the filtrate, $3 \mathrm{ml}$ was transferred into a test tube, added $3 \mathrm{ml}$ of TBA solution $(0.02 \mathrm{M})$ and mixed. After maintained in a water bath at $100{ }^{\circ} \mathrm{C}$ for 40 minutes, the test tubes were cooled in cold water for 5 minutes. Following centrifugation $(2000 \mathrm{~g}$, $5 \mathrm{~min}$ ), absorbance values were read at $530 \mathrm{~nm}$ using a spectrophotometer (Aquamate Thermo-Electron Corporation, England). The standard was prepared using TEP (1,1,3,3, tetraethoxypropane) and the $\mathrm{k}$ value was calculated as 0.06. Results are given in $\mu$ molmalonaldehyde $/ \mathrm{kg}$ (Lemon, 1975).

TBARS $=(($ absorbance $/ \mathrm{k}(0.06) \times 2 / 1000) \times 6.8) \times 1000 /$ sample weight 
The colour intensities $\left(\mathrm{L}^{*}, \mathrm{a}^{*}, \mathrm{~b}^{*}\right)$ of the cross sections of the samples were measured using a Minolta (CR-200, Minolta Co, Osaka, Japan) colorimeter.

The microbiological analyses of the samples were performed as described by Baumgart et al. (1993). Accordingly, $25 \mathrm{~g}$ of the meat samples was homogenized in $225 \mathrm{ml}$ of sterile Ringer's solution. Bacterial counts were expressed in log cfu g-1. Subsequently, the other solutions were prepared. Inoculations were made using the spread plate technique. The TMAB count was determined using Plate Count Agar (PCA, Merck). The petri dishes were incubated under aerobic conditions at $30 \pm 1{ }^{\circ} \mathrm{C}$ for $72+1$ hours. The TPAB count was also determined using the Plate Count Agar (PCA, Merck), and the petri dishes were incubated under aerobic conditions at $7 \pm 1^{\circ} \mathrm{C}$ for 10 days. For the determination of Enterobacteriaceae counts, $0.1 \mathrm{ml}$ of the appropriate dilutions was inoculated into Violet Red Bile Dextrose Agar (VRBDA, Merck). The petri dishes were incubated at $30{ }^{\circ} \mathrm{C}$ under anaerobic conditions for 2 days. Micrococcus/Staphylococcus counts were determined using mannitol-salt agar (MSA). The plates were incubated under aerobic conditions at $30 \pm 1^{\circ} \mathrm{C}$ for $48 \pm 1$ hours. $S$. aureus counts were determined using Baird Parker Agar (BPA) (Oxoid CM 275) supplemented with egg yolktellurite emulsion (Oxoid SR 54). The petri dishes were incubated under aerobic conditions at $37^{\circ} \mathrm{C}$ for 48 hours (Barbut, 2002). Bright black coloured catalase (+), oxidase (-) and coagulase (+) colonies with transparent zones that grew on BPA were evaluated to belong to $S$. aureus. Yeast and mold counts were determined using Rose Bengal Chloramphenicol (RBC) agar. The petri dishes were incubated under aerobic conditions at $25 \pm 1^{\circ} \mathrm{C}$ for 5 days.

\subsection{Statistical analysis}

Water activity, $\mathrm{pH}$, TBARS, colour parameters $\left(\mathrm{L}^{*}, \mathrm{a}^{*}\right.$ and $\left.\mathrm{b}^{*}\right)$ and microbial counts (logcfu g-1) were analysed using general linear model:

Yijk $=\mu+\mathrm{di}+\mathrm{gj}+\mathrm{dgij}+\mathrm{eijk}$

Yijk $=\mu+d i+g j+d g i j+e i j k$

WhereYijk $=$ response variable, $\mu=$ population mean, $d i=$ Storage Time (1., 3., 5., 7.,9. and 11. Days) (Water activity, $\mathrm{pH}$, TBARS, $\mathrm{L}^{*}, \mathrm{a}^{*}, \mathrm{~b}^{*}$, microorganism)], gj = treatment group (Control, YSPI, YSPII), dgij = Storage Time $\mathrm{x}$ treatment group interaction, eijk= experimental error. All statistical analyses were performed using the SPSS 10.00 software (SPSS, 1996).

\section{Results}

Thefindings obtained in the present study demonstrated a significant effect of Yucca schidigera powder on the water activity of the Musculus longissimus dorsi, while the effect of Yucca on the water activity of the Musculus gluteo biceps was found to be limited (Table 2). The results obtained in the present study have shown that both the Yucca schidigera powder and the length of the storage period of meat have significant effect on the $\mathrm{pH}$ values of the gluteo biceps and longissimus dorsi muscles (Table 2).

In the present study, it was observed that the antioxidant substances found in the structure of YSP, which was incorporated into the feed ration, eliminated free radicals from the environment, and thereby, significantly reduced lipid peroxidation in the Musculus gluteo biceps and Musculus longissimus dorsi (Table 2).

It was ascertained that, of the colour parameters of the Musculus gluteo biceps, the oxidation of $\mathrm{L}^{*}$ was affected variably by YSP, while $b^{*}$ was affected significantly by ST. This study showed that, in lambs, the oxidation of the colour parameters $\left(\mathrm{L}^{*}, \mathrm{a}^{*}, \mathrm{~b}^{*}\right)$ of the Musculus longissimus dorsi was significantly affected by ST. Furthermore, it was ascertained that the oxidation of $\mathrm{L}^{*}$ and $\mathrm{a}^{*}$ was significantly affected by the supplementation of the ration with YSP, and that the oxidation of $\mathrm{L}^{*}$ and $\mathrm{b}^{*}$ was significantly affected by T*ST (Table 3).

YSP significantly reduces the growth of Enterobacteriaceae and total mesophilic aerobic bacteria in the longissimus dorsi and gluteobiceps muscles, and thus, exhibits antimicrobial affect. On the other hand, YSP has been observed to reduce the growth of total mesophilic aerobic bacteria (TMAB) and Micrococcus/ Staphylococcus on certain days and to have no effect on their growth on some other days (Table 4 and 5). The supplementation of lamb rations with Yucca schidigera powder resulted in a significant increase in the yeast and mold counts of the musculus longissimus dorsi, but induced a variable effect on the musculus gluteo biceps, the variability of which was found to be statistically insignificant (Table 4 and 5). 
Table 1. Ingredients of crude nutrient proportions in the basal ration, $\%$.

\begin{tabular}{|c|c|c|c|}
\hline \multirow{2}{*}{ Item\% } & \multicolumn{3}{|c|}{ Groups } \\
\hline & Control & YSPI & YSPII \\
\hline Barley & 30 & 30 & 30 \\
\hline Maize & 20 & 20 & 20 \\
\hline Sunflower meal & 13.33 & 13.33 & 13.33 \\
\hline Cottonseed meal & 13.2 & 13.2 & 13.2 \\
\hline Wheatshorts & 9.70 & 9.69 & 9.68 \\
\hline Maizegluten & 5 & 5 & 5 \\
\hline DDGS $^{1}$ & 5 & 5 & 5 \\
\hline Marblepowder & 2.05 & 2.05 & 2.05 \\
\hline Molasses & 1.12 & 1.12 & 1.12 \\
\hline Salt & 0.5 & 0.5 & 0.5 \\
\hline Vitamin-mineral premix ${ }^{2}$ & 0.1 & 0.1 & 0.1 \\
\hline Yucca schidigera powder ${ }^{3}$ & - & 0.01 & 0.02 \\
\hline \multicolumn{4}{|c|}{ Analysed nutrient contents, $\%$} \\
\hline Crudeprotein & 18.53 & 18.52 & 18.52 \\
\hline Crudefat & 2.87 & 2.87 & 2.87 \\
\hline Crudefibre & 12.74 & 12.74 & 12.74 \\
\hline
\end{tabular}

${ }^{1}$ DDGS: Dried Distillers Grains with Solubles.

${ }^{2}$ The vitamin \& mineral premix provided the following (per $\mathrm{kg}$ ): 4,000,000 IU vitamin A, 800,000 IU vitamin D3, 5,000 IU vitamin E, $400 \mathrm{mg}$ vitamin B2, $2 \mathrm{mg}$ vitamin B12, 5,000 mg vitamin PP, 1,000 mg Dpantothenicacid, 20,000 mg choline, $50 \mathrm{mg} \mathrm{Co,} \mathrm{5,400} \mathrm{mg} \mathrm{Fe,} 185 \mathrm{mg} \mathrm{I}$, 6,900 mg Mn, $800 \mathrm{mg} \mathrm{Cu}, 6,400 \mathrm{mg}$ Zn, 14 mg Se.

${ }^{3}$ Exomix Yucca powder: $120 \mathrm{mg}$ of 1 kilogram of yucca includes (Ekol Food and Agriculture Livestock Marketing Industry and Trade Inc.).

Table 2. The effect of group and store period on water activity, pH and TBARS in lambs Musculus longissimus dorsi and Musculus gluteo biceps.

\begin{tabular}{llllllll}
\hline & \multirow{2}{*}{ Treatments } & \multicolumn{3}{c}{ Musculus gluteo biceps } & \multicolumn{2}{c}{ Musculus longissimus dorsi } \\
\cline { 2 - 7 } & & Wateractivity & pH & TBARS & Wateractivity & pH & TBARS \\
\hline 1 & Control & $0.985 \pm 0.001$ & $5.69 \pm 0.09$ & $2.24 \pm 0.85$ & $0.985 \pm 0.001$ & $5.69 \pm 0.12$ & $2.82 \pm 0.80$ \\
& YSPI & $0.985 \pm 0.001$ & $5.66 \pm 0.12$ & $1.33 \pm 0.36$ & $0.985 \pm 0.001$ & $5.62 \pm 0.07$ & $1.51 \pm 0.27$ \\
& YSPII & $0.986 \pm 0.001$ & $5.74 \pm 0.06$ & $1.6 \pm 0.33$ & $0.985 \pm 0.000$ & $5.73 \pm 0.09$ & $1.81 \pm 0.12$ \\
& Control & $0.986 \pm 0.001$ & $5.49 \pm 0.04$ & $3.51 \pm 1.36$ & $0.985 \pm 0.000$ & $5.48 \pm 0.04$ & $4.37 \pm 1.41$ \\
& YSPI & $0.986 \pm 0.003$ & $5.47 \pm 0.03$ & $2.73 \pm 1.13$ & $0.985 \pm 0.000$ & $5.46 \pm 0.02$ & $3.37 \pm 1.04$ \\
& YSPII & $0.986 \pm 0.001$ & $5.61 \pm 0.06$ & $3.65 \pm 1.25$ & $0.985 \pm 0.000$ & $5.58 \pm 0.04$ & $4.34 \pm 0.86$ \\
& Control & $0.984 \pm 0.002$ & $5.62 \pm 0.11$ & $11.11 \pm 3.94$ & $0.985 \pm 0.001$ & $5.57 \pm 0.02$ & $14.48 \pm 1.87$ \\
& YSPI & $0.985 \pm 0.000$ & $5.58 \pm 0.08$ & $6.95 \pm 1.83$ & $0.988 \pm 0.001$ & $5.51 \pm 0.01$ & $6.99 \pm 1.74$ \\
& YSPII & $0.986 \pm 0.001$ & $5.67 \pm 0.05$ & $6.19 \pm 2.33$ & $0.986 \pm 0.000$ & $5.66 \pm 0.05$ & $7.06 \pm 2.36$ \\
& Control & $0.986 \pm 0.001$ & $5.67 \pm 0.18$ & $16.06 \pm 5.19$ & $0.986 \pm 0.001$ & $5.52 \pm 0.07$ & $20.32 \pm 2.50$ \\
& YSPI & $0.986 \pm 0.001$ & $5.50 \pm 0.07$ & $11.93 \pm 1.67$ & $0.986 \pm 0.001$ & $5.45 \pm 0.04$ & $12.23 \pm 2.10$ \\
& YSPII & $0.986 \pm \pm 0.001$ & $5.67 \pm 0.12$ & $12.26 \pm 2.31$ & $0.985 \pm 0.001$ & $5.57 \pm 0.07$ & $13.30 \pm 2.97$ \\
9 & Control & $0.987 \pm 0.001$ & $5.67 \pm 0.12$ & $23.42 \pm 5.13$ & $0.989 \pm 0.000$ & $5.66 \pm 0.18$ & $27.70 \pm 2.93$ \\
& YSPI & $0.990 \pm 0.003$ & $5.68 \pm 0.13$ & $20.88 \pm 2.99$ & $0.990 \pm 0.002$ & $5.63 \pm 0.17$ & $21.41 \pm 3.70$ \\
& YSPII & $0.991 \pm 0.001$ & $5.72 \pm 0.12$ & $19.83 \pm 5.82$ & $0.991 \pm 0.001$ & $5.78 \pm 0.10$ & $25.25 \pm 1.00$ \\
& Control & $0.990 \pm 0.002$ & $5.77 \pm 0.13$ & $38.47 \pm 17.24$ & $0.990 \pm 0.000$ & $5.70 \pm 0.15$ & $53.13 \pm 11.65$ \\
& YSPI & $0.990 \pm 0.002$ & $5.61 \pm 0.14$ & $32.70 \pm 9.90$ & $0.991 \pm 0.002$ & $5.53 \pm 0.11$ & $41.45 \pm 5.31$ \\
& YSPII & $0.990 \pm 0.001$ & $5.76 \pm 0.13$ & $29.27 \pm 9.55$ & $0.992 \pm 0.001$ & $5.66 \pm 0.06$ & $37.37 \pm 4.06$ \\
$P$-value & ST & 0.000 & 0.000 & 0.000 & 0.000 & 0.000 & 0.000 \\
& T & 0.098 & 0.000 & 0.000 & 0.040 & 0.001 & 0.000 \\
& ST*T & 0.334 & 0.546 & 0.425 & 0.000 & 0.967 & 0.000 \\
\hline
\end{tabular}

pH: acidification, TBRAS: thiobarbituric acid-reactive substances,

Control: basal ration alone, YSPI: basal ration+100 ppm of Yucca schidigera powder, YSPII: basal ration+200 mg/kg of Yucca schidigera powder. ST: storage time, T: treatments. 
Table 3. The effect of group and store period on colour parameter [ $L$ (relativelightness), $\mathbf{a}^{*}$ (relativeredness), $\mathbf{b}^{*}$ (relativeyellowness)] in lambs Musculus longissimus dorsi and Musculus gluteo biceps.

\begin{tabular}{|c|c|c|c|c|c|c|c|}
\hline \multirow{2}{*}{ Days } & \multirow{2}{*}{ Treatments } & \multicolumn{3}{|c|}{ Musculus gluteo biceps } & \multicolumn{3}{|c|}{ Musculuss longissimus dorsi } \\
\hline & & $\mathbf{L}^{*}$ & a* & $\mathbf{b}^{*}$ & $\mathbf{L}^{*}$ & a* & $\mathbf{b}^{*}$ \\
\hline \multirow[t]{3}{*}{1} & Control & $44.32 \pm 3.32$ & $17.04 \pm 2.03$ & $6.04 \pm 2.37$ & $44.85 \pm 2.41$ & $15.80 \pm 1.25$ & $6.31 \pm 1.55$ \\
\hline & YSPI & $44.06 \pm 3.23$ & $15.98 \pm 1.68$ & $4.92 \pm 1.74$ & $44.55 \pm 1.62$ & $18.76 \pm 3.66$ & $9.10 \pm 1.88$ \\
\hline & YSPII & $46.17 \pm 2.32$ & $16.63 \pm 1.59$ & $6.77 \pm 1.71$ & $44.74 \pm 3.63$ & $15.89 \pm 2.91$ & $7.37 \pm 1.55$ \\
\hline \multirow[t]{3}{*}{3} & Control & $43.56 \pm 2.24$ & $14.40 \pm 2.72$ & $8.04 \pm 2.79$ & $44.86 \pm 3.20$ & $14.67 \pm 1.58$ & $8.16 \pm 1.89$ \\
\hline & YSPI & $44.64 \pm 2.61$ & $14.57 \pm 1.53$ & $7.95 \pm 2.23$ & $47.29 \pm 2.79$ & $15.44 \pm 1.90$ & $10.19 \pm 1.37$ \\
\hline & YSPII & $48.18 \pm 2.37$ & $17.33 \pm 3.04$ & $10.17 \pm 3.90$ & $47.17 \pm 2.10$ & $17.64 \pm 2.62$ & $9.21 \pm 2.79$ \\
\hline \multirow[t]{3}{*}{5} & Control & $43.30 \pm 3.20$ & $16.63 \pm 3.19$ & $4.86 \pm 2.11$ & $46.83 \pm 3.43$ & $14.00 \pm 2.31$ & $8.03 \pm 1.81$ \\
\hline & YSPI & $44.00 \pm 2.43$ & $16.05 \pm 2.33$ & $6.13 \pm 2.01$ & $46.47 \pm 2.31$ & $17.21 \pm 2.51$ & $9.23 \pm 2.74$ \\
\hline & YSPII & $45.95 \pm 4.58$ & $14.98 \pm 2.71$ & $6.52 \pm 1.16$ & $46.97 \pm 3.65$ & $14.63 \pm 1.52$ & $8.19 \pm 1.87$ \\
\hline \multirow[t]{3}{*}{7} & Control & $43.96 \pm 2.27$ & $15.38 \pm 2.89$ & $5.69 \pm 2.00$ & $44.69 \pm 1.70$ & $13.48 \pm 2.18$ & $9.53 \pm 2.96$ \\
\hline & YSPI & $45.65 \pm 2.36$ & $13.26 \pm 3.57$ & $8.88 \pm 3.41$ & $46.10 \pm 3.13$ & $14.98 \pm 2.21$ & $9.69 \pm 2.59$ \\
\hline & YSPII & $46.81 \pm 4.43$ & $14.84 \pm 3.85$ & $5.21 \pm 1.46$ & $49.46 \pm 4.13$ & $14.41 \pm 4.67$ & $8.34 \pm 1.99$ \\
\hline \multirow[t]{3}{*}{9} & Control & $47.10 \pm 3.36$ & $15.53 \pm 2.74$ & $6.28 \pm 2.39$ & $46.01 \pm 3.05$ & $12.57 \pm 3.79$ & $8.83 \pm 1.36$ \\
\hline & YSPI & $42.24 \pm 3.95$ & $15.73 \pm 3.77$ & $5.92 \pm 1.81$ & $47.56 \pm 2.63$ & $16.73 \pm 8.75$ & $10.04 \pm 2.54$ \\
\hline & YSPII & $44.84 \pm 3.02$ & $16.06 \pm 3.77$ & $6.42 \pm 2.24$ & $47.88 \pm 4.02$ & $15.81 \pm 3.64$ & $7.84 \pm 3.14$ \\
\hline \multirow[t]{3}{*}{11} & Control & $43.63 \pm 2.23$ & $15.64 \pm 3.33$ & $6.39 \pm 1.29$ & $45.26 \pm 1.68$ & $12.56 \pm 4.04$ & $10.66 \pm 2.02$ \\
\hline & YSPI & $45.89 \pm 2.53$ & $15.09 \pm 3.88$ & $6.73 \pm 2.65$ & $47.24 \pm 2.93$ & $14.38 \pm 2.74$ & $9.44 \pm 4.16$ \\
\hline & YSPII & $45.61 \pm 3.43$ & $15.34 \pm 4.22$ & $7.16 \pm 3.01$ & $48.98 \pm 4.58$ & $11.63 \pm 2.20$ & $12.33 \pm 2.62$ \\
\hline$P-$ & ST & 0.653 & 0.114 & 0.000 & 0.003 & 0.000 & 0.000 \\
\hline \multirow[t]{2}{*}{ value } & $\mathrm{T}$ & 0.000 & 0.278 & 0.104 & 0.000 & 0.000 & 0.300 \\
\hline & $\mathrm{ST} * \mathrm{~T}$ & 0.002 & 0.405 & 0.003 & 0.006 & 0.203 & 0.011 \\
\hline
\end{tabular}

$\mathrm{L}^{*}$ : relative lightness, $\mathrm{a}^{*}$ : relative redness, $\mathrm{b}^{*}$ : relative yellowness

Control: basal ration alone, YSPI: basal ration+100 ppm of Yucca schidigera powder, YSPII: basal ration $+200 \mathrm{mg} / \mathrm{kg}$ of Yucca schidigera powder. ST: storage time, T: treatments.

Table 4. The effects of group and storage time on Enterobacteriaceae, Total psychrotrophic aerobic bacteria (TPAB), Total mesophilic aerobic bacteria (TMAB), Micrococcus/Staphylococcus, Staphylococcusaureus (S. aureus) and yeast and mould counts in lambs Musculus gluteo biceps.

\begin{tabular}{llllllll}
\hline Days & Treatments & Enterobacteriaceae & TPAB & TMAB & $\begin{array}{l}\text { Micrococcus/ } \\
\text { Staphylococcus }\end{array}$ & S. aureus & $\begin{array}{l}\text { Yeast and } \\
\text { mold }\end{array}$ \\
\hline 1 & Control & $3.49 \pm 0.11$ & $0.70 \pm 0.00$ & $4.75 \pm 0.06$ & $4.58 \pm 0.21$ & $0.70 \pm 0.00$ & $2.55 \pm 0.26$ \\
& YSPI & $1.06 \pm 0.62$ & $0.70 \pm 0.00$ & $3.74 \pm 0.04$ & $2.70 \pm 0.70$ & $0.70 \pm 0.00$ & $2.72 \pm 0.05$ \\
& YSPII & $1.83 \pm 0.99$ & $0.70 \pm 0.00$ & $4.27 \pm 0.26$ & $2.78 \pm 0.14$ & $0.70 \pm 0.00$ & $2.43 \pm 0.19$ \\
3 & Control & $3.63 \pm 0.10$ & $3.91 \pm 0.07$ & $3.70 \pm 0.19$ & $3.61 \pm 0.25$ & $0.70 \pm 0.00$ & $3.15 \pm 0.07$ \\
& YSPI & $2.85 \pm 0.14$ & $0.70 \pm 0.00$ & $3.15 \pm 0.14$ & $3.48 \pm 0.41$ & $0.70 \pm 0.00$ & $3.11 \pm 0.16$ \\
& YSPII & $3.28 \pm 0.16$ & $0.70 \pm 0.00$ & $3.79 \pm 0.11$ & $3.74 \pm 0.14$ & $0.70 \pm 0.00$ & $3.40 \pm 0.12$ \\
5 & Control & $4.50 \pm 0.04$ & $4.60 \pm 0.13$ & $4.96 \pm 0.07$ & $4.02 \pm 0.02$ & $0.70 \pm 0.00$ & $3.52 \pm 0.15$ \\
& YSPI & $3.10 \pm 0.03$ & $4.72 \pm 0.09$ & $4.71 \pm 0.03$ & $3.76 \pm 0.25$ & $0.70 \pm 0.00$ & $3.85 \pm 0.06$ \\
& YSPII & $4.33 \pm 0.17$ & $4.49 \pm 0.10$ & $4.39 \pm 0.08$ & $4.09 \pm 0.05$ & $0.70 \pm 0.00$ & $3.84 \pm 0.07$ \\
7 & Control & $4.83 \pm 0.13$ & $6.11 \pm 0.65$ & $6.08 \pm 0.08$ & $4.18 \pm 0.09$ & $2.07 \pm 0.10$ & $4.21 \pm 0.17$ \\
& YSPI & $4.71 \pm 0.20$ & $5.89 \pm 0.49$ & $5.26 \pm 0.04$ & $4.54 \pm 0.28$ & $2.13 \pm 0.23$ & $4.19 \pm 0.04$ \\
& YSPII & $4.73 \pm 0.18$ & $6.42 \pm 0.66$ & $6.76 \pm 0.06$ & $4.65 \pm 0.17$ & $2.70 \pm 0.35$ & $4.18 \pm 0.04$ \\
9 & Control & $5.15 \pm 0.05$ & $5.88 \pm 0.05$ & $6.40 \pm 0.01$ & $4.50 \pm 0.02$ & $3.22 \pm 0.12$ & $5.39 \pm 0.25$ \\
& YSPI & $5.05 \pm 0.02$ & $5.67 \pm 0.06$ & $6.41 \pm 0.10$ & $4.77 \pm 0.17$ & $3.16 \pm 0.15$ & $4.81 \pm 0.06$ \\
& YSPII & $5.13 \pm 0.09$ & $6.40 \pm 0.06$ & $7.45 \pm 0.22$ & $4.87 \pm 0.13$ & $3.54 \pm 0.05$ & $4.63 \pm 0.17$ \\
& Control & $5.45 \pm 0.29$ & $6.16 \pm 0.08$ & $9.13 \pm 0.11$ & $5.40 \pm 0.22$ & $3.44 \pm 0.21$ & $5.90 \pm 0.02$ \\
& YSPI & $5.34 \pm 0.09$ & $8.02 \pm 0.58$ & $9.64 \pm 0.07$ & $5.16 \pm 0.15$ & $3.70 \pm 0.01$ & $5.53 \pm 0.16$ \\
\multirow{4}{*}{$P$-value } & YSPII & $6.28 \pm 0.22$ & $7.90 \pm 0.80$ & $10.24 \pm 0.09$ & $5.21 \pm 0.03$ & $3.65 \pm 0.05$ & $5.57 \pm 0.32$ \\
& ST & 0.000 & 0.000 & 0.000 & 0.000 & 0.000 & 0.000 \\
& T & 0.000 & 0.065 & 0.000 & 0.002 & 0.000 & 0.088 \\
& ST*T & 0.000 & 0.000 & 0.000 & 0.000 & 0.000 & 0.000 \\
\hline
\end{tabular}

Control: basal ration alone, YSPI: basal ration+100 ppm of Yucca schidigera powder, YSPII: basal ration+200 mg/kg of Yucca schidigera powder. ST: storage time, T: treatments. 
Table 5. The effects of group and storage time on Enterobacteriaceae, Total psychrotrophic aerobic bacteria (TPAB), Total mesophilic aerobic bacteria (TMAB), Micrococcus/Staphylococcus, Staphylococcus aureus (S. aureus) and yeast and mold counts in lambs Musculus longissimus dorsi.

\begin{tabular}{|c|c|c|c|c|c|c|c|}
\hline Days & Treatments & Enterobacteriaceae & (TPAB) & (TMAB) & $\begin{array}{l}\text { Micrococcus- } \\
\text { Staphylococcus }\end{array}$ & S. aureus & $\begin{array}{l}\text { Yeast and } \\
\text { mold }\end{array}$ \\
\hline \multirow[t]{3}{*}{1} & Control & $3.64 \pm 0.19$ & $0.70 \pm 0.00$ & $4.91 \pm 0.18$ & $3.21 \pm 0.31$ & $0.70 \pm 0.00$ & $2.83 \pm 0.10$ \\
\hline & YSPI & $0.80 \pm 0.17$ & $0.70 \pm 0.00$ & $3.93 \pm 0.22$ & $3.71 \pm 0.20$ & $0.70 \pm 0.00$ & $2.52 \pm 0.23$ \\
\hline & YSPII & $1.63 \pm 0.95$ & $0.70 \pm 0.00$ & $4.34 \pm 0.18$ & $3.49 \pm 0.13$ & $0.70 \pm 0.00$ & $2.51 \pm 0.16$ \\
\hline \multirow[t]{3}{*}{3} & Control & $2.62 \pm 0.13$ & $3.75 \pm 0.09$ & $4.06 \pm 0.42$ & $3.70 \pm 0.08$ & $0.70 \pm 0.00$ & $3.37 \pm 0.20$ \\
\hline & YSPI & $2.57 \pm 0.23$ & $0.70 \pm 0.00$ & $3.53 \pm 0.43$ & $3.71 \pm 0.20$ & $0.70 \pm 0.00$ & $3.37 \pm 0.29$ \\
\hline & YSPII & $2.63 \pm 0.29$ & $0.70 \pm 0.00$ & $3.82 \pm 0.11$ & $3.73 \pm 0.09$ & $0.70 \pm 0.00$ & $4.02 \pm 0.72$ \\
\hline \multirow[t]{3}{*}{5} & Control & $4.64 \pm 0.93$ & $5.61 \pm 0.03$ & $5.43 \pm 0.51$ & $4.50 \pm 0.09$ & $0.70 \pm 0.00$ & $3.77 \pm 0.17$ \\
\hline & YSPI & $3.55 \pm 0.15$ & $4.65 \pm 0.06$ & $5.15 \pm 0.48$ & $4.81 \pm 0.20$ & $0.70 \pm 0.00$ & $3.86 \pm 0.10$ \\
\hline & YSPII & $4.43 \pm 0.29$ & $5.21 \pm 0.02$ & $5.02 \pm 0.70$ & $4.16 \pm 0.20$ & $0.70 \pm 0.00$ & $3.90 \pm 0.08$ \\
\hline \multirow[t]{3}{*}{7} & Control & $5.07 \pm 0.05$ & $7.18 \pm 6.65$ & $6.38 \pm 0.35$ & $4.65 \pm 0.04$ & $2.71 \pm 0.24$ & $4.36 \pm 0.20$ \\
\hline & YSPI & $4.23 \pm 0.11$ & $6.09 \pm 0.48$ & $5.45 \pm 0.23$ & $4.93 \pm 0.15$ & $2.67 \pm 0.27$ & $4.58 \pm 0.20$ \\
\hline & YSPII & $5.15 \pm 0.08$ & $6.40 \pm 0.05$ & $6.63 \pm 0.24$ & $4.67 \pm 0.14$ & $2.78 \pm 0.26$ & $4.74 \pm 0.08$ \\
\hline \multirow[t]{3}{*}{9} & Control & $5.16 \pm 0.10$ & $6.85 \pm 0.02$ & $6.99 \pm 0.65$ & $4.90 \pm 0.07$ & $3.40 \pm 0.10$ & $4.75 \pm 0.18$ \\
\hline & YSPI & $5.72 \pm 0.16$ & $6.33 \pm 0.07$ & $6.78 \pm 0.42$ & $5.06 \pm 0.07$ & $3.30 \pm 0.07$ & $4.91 \pm 0.08$ \\
\hline & YSPII & $5.74 \pm 0.15$ & $6.76 \pm 0.06$ & $7.61 \pm 0.24$ & $5.37 \pm 0.09$ & $3.51 \pm 0.11$ & $4.93 \pm 0.05$ \\
\hline \multirow[t]{3}{*}{11} & Control & $6.25 \pm 0.18$ & $8.58 \pm 0.51$ & $8.92 \pm 0.53$ & $5.28 \pm 0.07$ & $3.73 \pm 0.13$ & $5.47 \pm 0.16$ \\
\hline & YSPI & $6.30 \pm 0.04$ & $8.06 \pm 0.04$ & $8.89 \pm 0.83$ & $5.17 \pm 0.08$ & $3.76 \pm 0.17$ & $5.58 \pm 0.17$ \\
\hline & YSPII & $6.63 \pm 0.14$ & $7.90 \pm 0.76$ & $9.22 \pm 1.12$ & $5.66 \pm 0.24$ & $3.73 \pm 0.10$ & $5.80 \pm 0.09$ \\
\hline \multirow[t]{3}{*}{$P$-value } & $\mathrm{ST}$ & 0.000 & 0.000 & 0.000 & 0.000 & 0.000 & 0.000 \\
\hline & $\mathrm{T}$ & 0.000 & 0.000 & 0.000 & 0.001 & 0.918 & 0.013 \\
\hline & $\mathrm{ST}^{*} \mathrm{~T}$ & 0.000 & 0.000 & 0.020 & 0.000 & 0.935 & 0.080 \\
\hline
\end{tabular}

Control: basal ration alone, YSPI: basal ration+100 ppm of Yucca schidigera powder, YSPII: basal ration+200 mg/kg of Yucca schidigera powder. ST: storage time,T: treatment.

\section{Discussion}

It is known that animal nutrition strategies enable the improvement of the quality and shelf life of meat. Thus, it has been reported that the supplementation of feed rations with antioxidants induces positive effects on meat quality and shelf life (Imik et al., 2010; Imik et al., 2012; Imik et al., 2012; Oral et al., 2014). Furthermore, the increasing demand of consumers for food containing natural supplements has drawn the attention to herbal additives. Several studies have been conducted on the effects of dietary supplementation with Yucca schidigera extract or powder on performance and metabolic parameters in animal production (Cheeke, 2009; Eryavuz and Dehority, 2004; Kutlu et al., 2001; Wang et al., 1998), yet no previous study exists on the effect of Yucca on meat quality and shelf life.

Post-mortem metabolism, which defines meat quality, is affected by both internal factors (water activity, natural antioxidants, antimicrobial substances and free radicals, etc.) and external factors (length of storage period, temperature and atmospheric pressure at which meat is stored and storage type, etc.).

It is known that water activity has a major role in the post-mortem metabolism of meat. The reduction of water activity to a minimum level is considered as a significant criterion for post-mortem metabolism and meat quality. Water activity values reflect the amount of water available for the use of microorganisms for their metabolism and performance. Therefore, water activity is considered a microbiological stability indicator for food and is attached great importance in food technology (Isengard, 2001). The findings of this study have shown that the impact of Yucca schidigera on the water activity of the Musculus longissimus dorsi is greater than that on the water activity of the Musculus gluteo biceps.

The conversion of glycogen into lactic acid and the reduction of the $\mathrm{pH}$ value in muscles both play an important role in the post-mortem metabolism of meat. Hopkins and Fogaty (Hopkins and Fogarty, 1998) reported that the $\mathrm{pH}$ value of lamb meat varied with breed and muscle (5.0-5.96), whilst Teixeira et al. (Teixeiraet al., 2005) reported variances with breed and slaughter weight (5.5-5.9). Yucca schidigera frequently contains saponins such as D-glucose, D-galactose, D-glucuronic acid, D-ribose, D-xylose, L-arabinose, L-fructose and L-ramnose as well as carbohydrates including 3-methyl glucose, quinovose and apiose (Mahato et al., 1998; Milgate and Roberts, 1995; Oleszek, 2002). The results obtained in the present study have shown that both the Yucca schidigera powder and the length of the storage period of meat have significant effect on the $\mathrm{pH}$ values of the gluteobiceps and longissimus dorsi muscles (Table 2), and the values obtained were determined to display 
similarity to the reference values indicated in literature (5.45-5.77) (Hopkins and Fogarty, 1998; Teixeira et al., 2005). These results have also demonstrated that the supplementation of feed rations with $100 \mathrm{ppm}$ of YSP significantly reduced muscle $\mathrm{pH}$ values and significantly affected glycogen rates and lactic acid formation. Low $\mathrm{pH}$ values having been measured in meat belonging to Group YSPI may be considered to arise from the carbohydrates found in the structure of saponins, yet high $\mathrm{pH}$ values having been measured in Group YSPII cannot be fully explained, and it is hypothesized that high saponin content adversely affects carbohydrate metabolism, and thus $\mathrm{pH}$ values.

Free radicals generated by metabolic processes in the organism bring about the occurrence of oxidative reactions. The first products of oxidative reactions are peroxides, and are followed by the formation of hydrocarbons, aldehydes, ketones, alcohols and organic acids. These products have adverse effects on the nutritional value, sensorial properties and shelf life of animal products (El-Massry et al., 2002). Antioxidants play an important role in the elimination of free radicals. It is well known that antioxidants significantly reduce lipid peroxidation, one of the major indicators of free radicals, in muscle tissue (Halici et al., 2012; Imik et al., 2010; Imik et al., 2012; Imik et al., 2012). Saponins and phenolic compounds found in the structure of Yucca schidigera (resveratrol, yuccaols A, B, C, D, E and yucaone A) have been shown to affect antioxidant activity positively owing to their redox property (Cheeke, 2009; Enginar et al., 2006). Aksu et al. (2007) reported that the phenolic compounds found in sorghum significantly reduce lipid oxidation and thereby, positively affect meat quality. Nasri et al. (2012) determined that the supplementation of lamb rations with Quillaja saponaria had no effect on lipid oxidation in the longissimus dorsi muscle.

In the present study, it was observed that the antioxidant substances found in the structure of YSP, which was incorporated into the feed ration, eliminated free radicals from the environment, and thereby, significantly reduced lipid peroxidation in the Musculus gluteo biceps and Musculus longissimus dorsi (Table 2). Yucca schidigera having determined to increase the water activity of the longissimus dorsi muscle, and at the same time, to reduce lipid peroxidation demonstrates that it contains highly effective antioxidant substances. Furthermore, the detection of a Days*Treatments interaction in the Musculus longissimus dorsi further supports this finding.

The natural colour of meat is formed by myoglobin and haemoglobin pigments. These pigments are composed of globin, a porphyrin ring and an iron ion. These three compounds, which define the colour of meat, are all rather sensitive to oxidation (Barbut, 2002; Mancini and Hunt, 2005). Teixeira et al. (2005) reported that, varying with body weight, sex and breed, the $\mathrm{L}^{*}, \mathrm{a}^{*}$ and $\mathrm{b}^{*}$ values of lamb meat ranged between $34.1-53.2$, 11.5-21.5 and 6.5-12.5, respectively. Sante'-Lhoutellier et al. (2008) determined that, in lamb meat, of the colour parameters, the $\mathrm{L}^{*}, \mathrm{a}^{*}$ and $\mathrm{b}^{*}$ values were not affected by roughage or concentrated feed but were influenced by the length of the storage period. Hopkins and Fogarty (1998) reported that the colour parameters of the $M$. longissimus thoracis varied with breed while no such variance was observed in the $M$. semimembranosus. Nasri et al. (2012) suggested that the supplementation of lamb rations with Quillaja saponaria contributed to the stability of the colour quality of the longissimus dorsi muscle. In the present study has been determined that, the oxidation of the colour parameters of muscles is directly proportional to the water activity of muscles.

Meat quality is inversely proportional to microbial population. It is known that microbial population increases directly proportional to prolonged storage and increased water activity. Antimicrobial substances found in the structure of meat play a major role in the preservation of meat. Previous research has shown that the shelf life of meat increases with the use of plant leaves and extracts containing substances with bactericidal and bacteriostatic effects (Bornez et al., 2009; Mastromatteo et al., 2010).

Researches are available, which suggest that the supplementation of ruminant rations with Yucca schidigera extract induces antiprotozoal and antimicrobial effect in the rumen, and thereby, regulates fermentation and enables the better utilisation of nutrients (Eryavuz and Dehority, 2004; Hu et al., 2005; Pen et al., 2006). The saponin content of Yucca schidigera has antiprotozoal effect. Saponins react with the cholesterol found in the cell membrane of protozoans, disrupt their cellular integrity, and thereby, cause the death of protozoa (Wang et al., 1998). However, no scientific literature is available on how the saponin content of YSP affects the microbiological quality of meat. The effects of Yucca schidigera powder on microbial growth in the Musculus gluteo biceps and Musculus longissimus dorsi are presented in Tables 4 and 5. Of these microorganisms, total mesophilic aerobic bacteria are used as an indicator of microbiological quality of food. In the present study, while it was observed that YSP had no effect on the growth of Staphylococcus aureus in the longissimus dorsi muscle, the counts of this bacterium was determined to have increased significantly in the gluteobiceps muscle on days 7, 9 and 11, in comparison to the control group. The significant increase detected in the water activity of the groups that received Yucca schidigera powder can be considered as the cause of microbial growth. The 
storage period of meat and microbial growth are directly proportional to each other. In the present study, it was observed that the antibacterial effect of Yucca schidigera powder on the longissimus dorsi and gluteobiceps muscles varied with bacterial species. Furthermore, these findings demonstrate that Yucca schidigera does not have antifungicidal property (Table 4 and 5).

\section{Conclusions}

In conclusion, the incorporation of YSP into lamb feed rations significantly increased the water activity of the longissimus dorsi muscle, but was observed to have limited effect on the water activity of the gluteobiceps muscle. In Group YSPI, the $\mathrm{pH}$ values of both muscles having been determined to be significantly reduced, demonstrated YSP to affect the glycogen metabolism of muscles. It was ascertained that YSP significantly reduced lipid peroxidation in the longissimus dorsi and gluteobiceps muscles. Furthermore, it was detected that YSP affected the colour parameters of the longissimus dorsi muscle more than that of the gluteobiceps muscle. While YSP was confirmed to have bactericidal effect, no antifungicidal effect was detected. Further studies are required to fully elucidate the effect of the supplementation of ruminant rations with Yucca schidigera on meat quality.

\section{Acknowledgements}

This study was performed on the gluteobiceps and longissimus dorsi muscles of animals slaughtered after the end of the trials conducted for the thesis titled "The effect of saponin (Yucca schidigera) added to lamb feed on fattening performance, rumen and some blood parameters, the immune system and the antioxidative metabolism of the hepatic tissue".

\section{Conflict of interest}

None to declare.

\section{References}

Aksu MI, H Imik and M Karaoglu, 2007. Influence of dietary sorghum (sorghum vulgare) and corn supplemented with methionine on cut-up pieces weights of broiler carcass and quality properties of breast and drumsticks meat. Food Sci.Tech. Int., 13: 361-367.

Alimentarius C, 2002. Codex Alimentarius Commission. In Available from:http://www.codexalimentarius.net/ gsfaonline/additives/index.htmlUpdated up to the 33rd Session of the Codex Alimentarius Commission.

AOAC, 2005. Official methods of analysis of AOAC International (18 ed.). USA: AOAC International.

Barbut S, 2002. Poultry products processing: An industry guide: CRC Press, Boca Raton, FL.

Baumgart J, J Firnhaber and G Spcher, 1993. Mikrobiologische untersuchung von lebensmitteln (Hamburg, Germany, Behr's Verlag).

Bórnez R, M Linares and H Vergara, 2009. Microbial quality and lipid oxidation of Manchega breed suckling lamb meat: Effect of stunning method and modified atmosphere packaging. Meat Sci., 83: 383-389.

Cheeke P, 2009. In Applications of saponins as feed additives in poultry production, 20 th Annual Australian Poultry Science Symposium, 50.

El-Massry KF, AH El-Ghorab and A Farouk, 2002. Antioxidant activity and volatile components of Egyptian Artemisia judaica L. Food Chemistry, 79: 331-336.

Enginar H, G Avci, A Eryavuz, E Kaya, I Kucukkurt and AF Fidan, 2006. Effect of Yucca schidigera extract on lipid peroxidation and antioxidant activity in rabbits exposed to gamma-radiation. Revue Med. Vet., 157: 415-419.

Eryavuz A and BA Dehority, 2004. Effect of Yucca schidigera extract on the concentration of rumen microorganisms in sheep. Anim. Feed Sci. Technol., 117: 215-222.

Gokalp H, M Kaya, Y Tulek and O Zorba, 2001. Guide for quality control and laboratory application of meat products (4th press). Atatürk Univ. Publ, No: 751, Erzurum, Turkey.

Halici M, H Imik, M Koc and R Gumus, 2012. Effects of a-lipoic acid, vitamins E and C upon the heat stress in Japanese quails. J. Anim. Physiol. Anim. Nutr., 96: 408-415.

Hopkins D and N Fogarty, 1998. Diverse lamb genotypes-2. Meat pH, colour and tenderness. Meat Sci., 49: 477-488.

Hu WL, JX Liu, JA Ye, YM Wu and YQ Guo, 2005. Effect of tea saponin on rumen fermentation in vitro. Anim. Feed Sci. Technol., 120: 333-339. 
Imik H, MA Atasever, M Koc, M Atasever and K Ozturan, 2010. Effect of dietary supplementation of some antioxidants on growth performance, carcass composition and breast meat characteristics in quails reared under heat stress. Czech J. Anim. Sci., 55: 209-220.

Imik H, MA Atasever, S Urcar, H Ozlu, R Gumus and M Atasever, 2012. Meat quality of heat stress exposed broilers and effect of protein and vitamin E. Br. Poult. Sci., 53: 689-698.

Imik H, H Ozlu, R Gumus, MA Atasever, S Urcar and M Atasever, 2012. Effects of ascorbic acid and $\alpha$-lipoic acid on performance and meat quality of broilers subjected to heat stress. Br. Poult. Sci., 53: 800-808.

Isengard HD, 2001. Water content, one of the most important properties of food. Food Cont., 12: 395-400.

Kutlu HR, M Gorgulu and I Unsal, 2001. Effects of dietary Yucca schidigera powder on performance and egg cholesterol content of laying hens. J. Appl. Anim. Res., 20: 49-56.

Lemon D, 1975. An improved TBA test for rancidity. New Series Circular No. 51. Environment Canada. Fisheries and Marine Service, Halifax, NS.

Mahato SB, SK Sarkar and G Poddar, 1988. Triterpenoid saponins. Phytochem., 27: 3037-3067.

Mancinii R and M Hunt, 2005. Current research in meat color. Meat Sci., 71: 100-121.

Mastromatteo M, A Danza, A Conte, G Muratore and MA Del Nobile, 2010. Shelf life of ready to use peeled shrimps as affected by thymol essential oil and modified atmosphere packaging. Int. J. Food Microbiol., 144: 250-256.

Milgate J and D Roberts, 1995. The nutritional \& biological significance of saponins. Nutrition Research., 15: 1223-1249.

Nasri S, G Luciano, V Vasta, D Aouadi, A Priolo, HPS Makkar and H Ben Salem, 2012. Effect of Quillaja saponaria dietary administration on colour, oxidative stability and volatile profile of muscle longissimus dorsi of Barbarine lamb. Meat Sci., 92: 582-586.

Oleszek W, 2002. Chromatographic determination of plant saponins. J. Chromatogr. A, 967: 147-162.

Oral Toplu HD, A Nazligül, S Karaarslan, M Kaya and O Yagın, 2014. Effects of heat conditioning and dietary ascorbic acid supplementation on growth performance, carcass and meat quality characteristics in heatstressed broilers. Ankara Üniv. Vet. Fak. Derg., 61: 295-302.

Pen B, C Sar, B Mwenya, K Kuwaki, R Morikawa and J Takahashi, 2006. Effects of Yucca schidigera and Quillaja saponaria extracts on in vitro ruminal fermentation and methane emission. Anim. Feed Sci. Techno., 129: 175-186.

Piacente S, P Montoro, W Oleszek and C Pizza, 2004. Yucca schidigera bark: phenolic constituents and antioxidant activity. J. Natural Products, 67: 882-885.

Santé-Lhoutellier V, E Engel and P Gatellier, 2008. Assessment of the influence of diet on lamb meat oxidation. Food Chem., 109: 573-579.

SPSS, 1996. Statistical Packages for the Social Sciences: SPSS for Windows release 10.01.In SPSS Inc Chicago, IL, USA.

Tanaka O, Y Tamura, H Masuda and K Mizutani, 1996. Application of saponins in foods and cosmetics: saponins of Mohave yucca and Sapindus mukurossi. Advances in Experimental Medicine and Biology, 405: 1-11.

Teixeira A, S Batista, R Delfa and V Cadavez, 2005. Lamb meat quality of two breeds with protected origin designation. Influence of breed, sex and live weight. Meat Sci., 71: 530-536.

Van Soest PJ and JB Robertson, 1985. Analysis of forages and fibrous foods, in: laboratory manual for animal science, 613, Department of Animal Science, Ithaca: Cornell University, pp. 202.

Wang Y, TA McAllister, CJ Newbold, LM Rode, PR Cheeke and KJ Cheng, 1998: Effects of Yucca schidigera extract on fermentation and degradation of steroidal saponins in the rumen simulation technique (RUSITEC). Anim. Feed Sci. Techno., 74: 143-153. 